\title{
From FOBt to FIT: making it work for patients and populations 㹰
}

\author{
Authors: Guy Mole, ${ }^{\mathrm{A}}$ John Withington ${ }^{\mathrm{B}}$ and Robert Logan ${ }^{\mathrm{C}}$
}

\section{Introduction}

Colorectal cancer (CRC) is the UK's third most common cancer with 41,804 cases and 16,384 deaths in 2016. ${ }^{1,2}$ Improving survival depends on diagnosis at an early stage which can most effectively be achieved within the general population by increasing uptake and coverage of the National Bowel Cancer Screening Programme (NBCSP).

For patients presenting to primary care symptom-based referral criteria have been broadened in an attempt to detect early stage disease, while National Institute for Health and Care Excellence (NICE) NG12 guidance introduced testing for the presence (or absence) of occult blood using guaiac based tests. ${ }^{3}$ These changes have led to more urgent 2-week-wait (2WW) cancer referrals however colonoscopies in this group do not detect CRC or other serious bowel pathology. ${ }^{4}$ In 2017, NICE recommended replacing the guaiac based test with the newer faecal immunochemical test (FIT) which is more specific for blood, ${ }^{5}$ however the demand for colonoscopy from $2 \mathrm{WW}$ referral remains a challenge. A 1-day conference at the Royal College of Physicians considered how to utilise FIT most effectively as a means of addressing these issues in the symptomatic population.

\section{Clinical biochemical considerations and NICE guidance}

In the first session, Sally Benton provided context from the lab highlighting pre-analytical challenges such as the heterogeneity of faeces, the potential significance of haemoglobin variant $5^{5}$ and the instability of haemoglobin in faeces. ${ }^{6}$ She also discussed analytical challenges around assay standardisation as there are four different FIT manufacturers (although only 3 are approved by NICE) and so without standardisation it is difficult to directly compare results from different studies (see Fig 1). A further problem is that labs are unable to get sufficient reference material for standardisation. However, the International Federation

Authors: Anational medical director's clinical fellow, NHS England, London, UK; ${ }^{B}$ national medical director's clinical fellow, NHS England, London, UK; C Consultant gastroenterologist, Department of Gastroenterology, King's College Hospital, London, UK

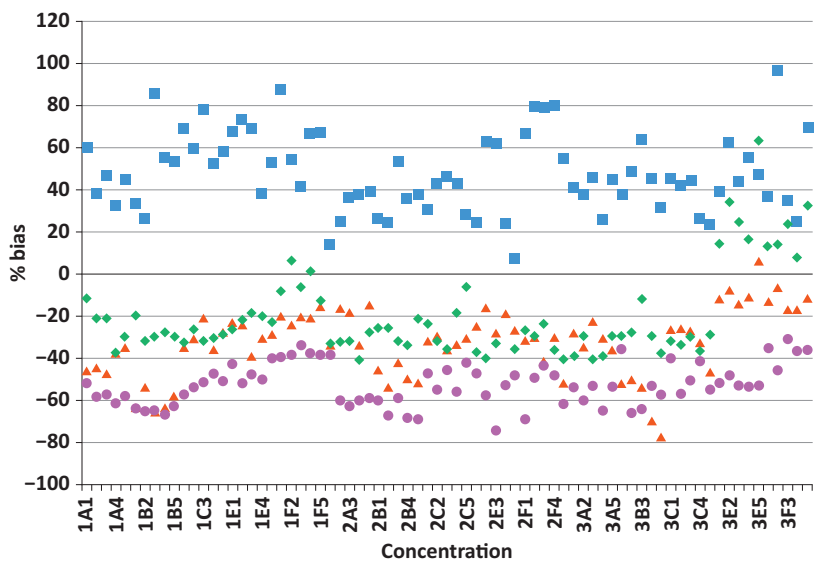

Fig 1. Results from external quality assessment material run on four different faecal immunochemical test analysers highlighting variation between instruments.

of Clinical Chemistry and Laboratory Medicine hope to have standardised FIT testing imminently. Another issue is the different limits of quantification ${ }^{7}$ while the key question is 'Should we be reporting down to the limit of detection and if not, what is the optimum diagnostic threshold?'

This was followed by an overview of the current NICE guidelines about the use of FIT in symptomatic patients. ${ }^{8}$ A major issue for DG30 related to the limited evidence base (only 10 studies on less than 7,000 patients) which could not be related directly to referral criteria as defined by NG12. In addition, most of the studies focused on the negative predictive value (NPV) of FIT to 'rule out' cancer in patients already referred for endoscopy. ${ }^{8}$ However, to ensure alignment with previous guidance, the final recommendation within DG30 focuses on the positive predictive value (PPV) of FIT as a 'rule-in' test. Within his presentation, Dr Logan explained how for most (low prevalent) conditions seen in primary care (and certainly for (RC) small changes to the prevalence can have a significant impact on the PPV. Therefore, by also lowering the referral threshold for suspected CRC within DG30, the PPV of FIT may be much lower than previously estimated (see Fig 2). This set the context for the next session which looked at how the NPV could potentially be used as a 'rule out' test in high risk patients. 


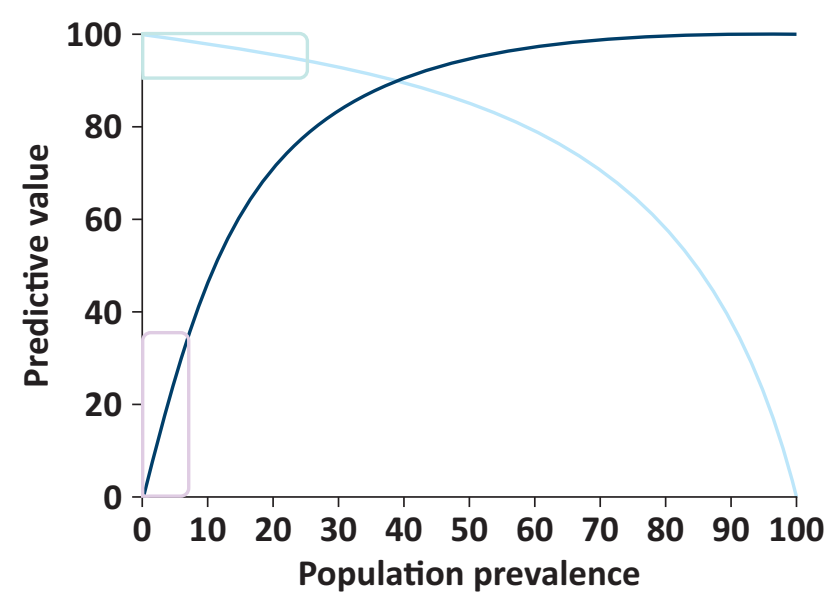

Fig 2. Relationship between negative (light blue line) and positive (dark blue line) predictive value with disease prevalence. The light teal box itlustrates focus of the evidence review ${ }^{4}$ and the light purple box illustrates the recommendation from National Institute for Health and Care Excellence. ${ }^{8}$

Implementing FIT in symptomatic populations: practice, learning and safety netting as a 'rule out' test

Six 'FIT pioneer' sites shared data from formal research studies and service evaluations where FIT is already being used in 2WW patient pathways.

Looking at the service evaluations, Nottingham has used FIT (OC-Sensor ${ }^{\mathrm{T} M}$ ) to triage patients to their first test since 2016. Patients with rectal bleeding are excluded and thresholds assessed at the limit of detection, $10 \mu \mathrm{g} / \mathrm{g}$ (as per NICE DG30) and $150 \mu \mathrm{g} / \mathrm{g}$. A FIT $<4 \mu \mathrm{g} / \mathrm{g}$ equated to a $0.2 \%$ risk of CRC while one between 4 and $10 \mu \mathrm{g} / \mathrm{g}$ equated to a $4.8 \%$ risk of CRC and these patients normally had computed tomography colonography (CTC) or colonoscopy as the first investigation. In Leicester, CTC is the primary imaging modality for all patients with change in bowel habit ${ }^{9}$ and a FIT $\left(\right.$ OC-Sensor $\left.{ }^{T M}\right)>4 \mu \mathrm{g} / \mathrm{g}$. Patients with rectal bleeding, weight loss, iron deficiency anaemia and abdominal or rectal mass are not included in their
FIT pathway. Lastly, results were reported from Eastbourne where they looked at 1,000 2WW referrals with NG12 symptoms. They used stool, collected during digital rectal examination, for FIT testing, with the result used to triage patients to the most appropriate imaging test. This study used HM-JACKarc ${ }^{T M}$ with a cut off at $10 \mu \mathrm{g} / \mathrm{g}$ and reported seven FIT negative cancers $(<1 \%$ of the total study population, see Table 1$)$. It was highlighted that in several of the studies, patients subsequently found to have CRC (but with an initial negative FIT test), had iron deficiency anaemia at presentation. It was suggested that the biological explanation for this may be due to degradation of haemoglobin from caecal tumours during colonic transit. This also highlighted the importance of a full blood count as part of safety netting in primary care.

Three on-going research trials are also assessing the NPV of FIT and there are plans to combine their data which would contain more than 20,000 patients. The quantitative FIT study in London is looking at all $2 \mathrm{WW}$ referrals using FIT (OC-Sensor ${ }^{\mathrm{TM}}$ ) and other aspects such as general practitioner (GP) acceptability. They have detected 76 cancers from 2,801 cases with nine FIT negative cancers at a cut-off of $10 \mu \mathrm{g} / \mathrm{g}$. Mr Abulafi from RM Partners highlighted how NICE had aimed to reduce the detection of CRC threshold to around $3 \%$ and that they had achieved this aim but with the trade-off of many more $2 \mathrm{WW}$ referrals. ${ }^{10}$ He reported on the largest study which has recruited 11,000 2WW patients and reports 11 FIT negative cancers at a cut-off of $10 \mu \mathrm{g} / \mathrm{g}$ and four at $2 \mu \mathrm{g} / \mathrm{g}$. Modelling showed a reduction in colonoscopy of $30-50 \%$ would be achieved while missing very few cancers. This may help to free up colonoscopy capacity to provide for the bowel cancer screening programme and opportunistic screening through DG30. Data from York, presented by Dr Turvill, highlighted even further the trade-off between trying not to miss any cancer (by using the lowest possible cut-off) versus optimising the use of colonoscopy by using a higher cut off.

\section{Implementing FIT in primary care / PPV}

The afternoon session looked at FIT in primary care based on its PPV with experience shared from three sites. The investigators in Nottingham having previously reported on their pilot work with FIT on the $2 \mathrm{WW}$ pathway ${ }^{11}$ have now introduced FIT for all $2 \mathrm{WW}$

Table 1. Demonstrates the number of faecal immunochemical test positive and negative cancers at different thresholds as well as the negative predictive value by faecal immunochemical test pioneer centre

\begin{tabular}{|c|c|c|c|c|c|c|}
\hline \multirow[b]{2}{*}{ Centre } & \multirow[b]{2}{*}{ n } & \multirow[b]{2}{*}{ FIT test } & \multirow[b]{2}{*}{ Threshold, $\mu \mathrm{g} \mathrm{Hb} / \mathrm{g}$ faeces } & \multicolumn{2}{|c|}{ Cancers detected } & \multirow[b]{2}{*}{ NPV, \% } \\
\hline & & & & FIT positive, n (\%) & FIT negative, $n(\%)$ & \\
\hline Eastbourne & 928 & HM-Jack ${ }^{T M}$ & 10 & $41(4.4)$ & $7(0.75)$ & 99.05 \\
\hline North London (qFIT pilot) & 2,801 & OC-Sensor ${ }^{\mathrm{TM}}$ & 10 & $76(2.7)$ & $9(0.32)$ & 99.4 \\
\hline \multirow[t]{2}{*}{ York 1} & 515 & HM-Jack ${ }^{T M}$ & $\geq 2$ (limit of detection) & $26(5.0)$ & $2(0.39)$ & 99.4 \\
\hline & & & $\geq 12$ & $24(4.7)$ & $4(0.78)$ & 99.1 \\
\hline \multirow[t]{3}{*}{ York 2} & 869 & HM-Jack $^{T M}$ & $\geq 2$ (limit of detection) & $12(1.4)$ & $1(0.12)$ & 99.8 \\
\hline & & & 10 & $11(1.3)$ & $2(0.23)$ & 99.7 \\
\hline & & & 44.5 & $11(1.3)$ & $2(0.23)$ & 99.7 \\
\hline \multirow[t]{2}{*}{ Croydon (NICE FIT study) } & 4,069 & HM-Jack ${ }^{T M}$ & 10 & $94(2.3)$ & $11(0.27)$ & 99.7 \\
\hline & & & 2 & $101(2.5)$ & $4(0.01)$ & 99.9 \\
\hline
\end{tabular}


Table 2. Key clinical, test and implementation questions

$\begin{array}{ll}\text { Clinical } & \text { How effective is FIT NPV as a rule out and can } \\ \text { it be improved? } & \text { How often does FIT miss cancers? } \\ & \text { Can a risk score be derived using the PPV? } \\ \text { Test } & \text { Is there a difference between the tests? } \\ & \text { What are appropriate cut-off levels? } \\ & \text { What instructions should be given to patients } \\ & \text { and general practitioners? } \\ \text { Implementation } & \text { Impact of FIT on endoscopic services? } \\ & \text { Acceptability to general practitioners and } \\ & \text { patients? } \\ & \text { What are the barriers? }\end{array}$

FIT = faecal immunochemical test; NPV = negative predictive value; PPV = positive predictive value.

referrals from primary care. They reported seeing a reduction in the use of colonoscopy but mainly due to an increase in the use of CTC. However, they have seen more cancers diagnosed on the 2WW pathway with some evidence of stage migration for more cancers to be diagnosed at stage 1 or 2 . Anaemia was shown to be a strong predictor for CRC and the PPV of FIT was improved by including haemoglobin or ferritin in a risk assessment. The conclusion was that FIT is better at determining patients for referral than symptoms with the trade-off that 62-day performance declined due to increased volume of referrals.

Dr Nicholson discussed the approach in Oxford where GPs have access to FIT for any patient, not only those referred on the $2 \mathrm{WW}$ pathway. He also concluded that FIT is better than symptoms for deciding who to refer for colonoscopy with fewer missed cancers and that GPs use the test judiciously. Their pathway introduced FIT (OC-Sensor ${ }^{\mathrm{TM}}$ ) in the low risk symptomatic population (DG30) with a cut off at $7 \mu \mathrm{g} / \mathrm{g}$ and the result reported as either positive or negative. ${ }^{12}$ The exception was for males and post-menopausal females with iron deficiency anaemia in which case referral for endoscopy was recommended. They reported 1,457 patients with a negative FIT so far and no cancers found in this group although only 201 have had colonoscopy. Dr Hunt from Lancashire also reported using FIT (OC-Sensor ${ }^{\mathrm{TM}}$ ) but with a cut off at $10 \mu \mathrm{g} / \mathrm{g}$ in the low risk symptomatic population (DG30). Prevalence of cancer in their cohort was $3.5 \%$ and all cancers found in the FIT positive group had a value from 75 to $608 \mu \mathrm{g} / \mathrm{g}$.

Prof Halloran explained that the quantitative value of FIT hints at the pathology and reiterated that the PPV provides an opportunity to create a predictive score, based on simple measures, such as age, gender or deprivation which is also known to be an important risk factor for $\mathrm{CRC} .^{13}$ While other groups have combined the quantitative properties of FIT with enhanced computer learning of serial full blood count to improve the PPV for non-symptom based risk scores for CRC.

\section{Evidence synthesis and next steps}

Natasha Crawford from Cancer Alliance Data, Evidence and Analysis Service at NHS England and Public Health England provided an oversight of the work being done by the 'FIT pioneer' sites. She concluded that there is emerging evidence that FIT is effective at ruling out CRC but important knowledge gaps remain (Table 2). She anticipated that as the pioneer group report their findings these gaps will be filled and allow NHS England guidance to be updated later this year.

\section{Conclusion}

FIT is a highly accurate quantitative test for detecting 'occult' haemoglobin in faeces. Its implementation in the NBCSP will improve uptake particularly in those populations most at risk of CRC. Maximising its value in the symptomatic population however will depend on how it is implemented within secondary and primary care. By collating data and sharing learning from the FIT pioneer sites, NHS England and its partners will be able to issue further guidance to support best practice at the earliest opportunity.

\section{Acknowledgements}

Aftab Khan, Ayan Banerjea, Baljit Singh, David Humes, James Turvill, Michael Machesney, Muti Abulafi, Natasha Crawford, Sally Benton, Stephen Halloran, Natalie Hunt, Brian Nicholson, Tim James and Michelle Barclay.

\section{References}

1 White A, Ironmonger L, Steele R et al. A review of sex-related differences in colorectal cancer incidence, screening uptake, routes to diagnosis, cancer stage and survival in the UK. BMC Cancer 2018;18:906.

2 Cancer Research UK. Bowel cancer statistics. Cancer Research UK, 2018. www.cancerresearchuk.org/health-professional/cancerstatistics/statistics-by-cancer-type/bowel-cancer [Accessed 03 July 2018].

3 National Institute for Health and Care Excellence. Suspected cancer: recognition and referral. NICE guideline [NG12]. London: NICE, 2015.

4 Westwood M, Lang S, Armstrong $\mathrm{N}$ et al. Faecal immunochemical tests (FIT) can help to rule out colorectal cancer in patients presenting in primary care with lower abdominal symptoms: a systematic review to inform NICE DG30 diagnostic guidance. BMC Med 2017;15:189.

5 Carroll M, John C, Mantio D, Djedovic N, Benton C. An assessment of the effect of haemoglobin variants on detection by faecal immunochemical tests. Ann Clin Biochem 2018;55:706-9.

6 Mellen S, de Ferrars M, Chapman C et al. Evaluation of sample stability for a quantitative faecal immunochemical test and comparison of two sample collection approaches. Ann Clin Biochem 2018;55:657-64.

7 Fraser C, Benton S. Detection capability of quantitative faecal immunochemical tests for haemoglobin (FIT) and reporting of low faecal haemoglobin concentrations. Clin Chem Lab Med 2018: in print.

8 National Institute for Health and Care Excellence. Quantitative faecal immunochemical tests to guide referral for colorectal cancer in primary care. Diagnostics guidance [DG30]. London: NICE, 2017.

9 Stephenson J, Pancholi J, Ivan C et al. Straight-to-test faecal tagging CT colonography for exclusion of colon cancer in symptomatic patients under the English 2-week-wait cancer investigation pathway: a service review. Clin Radiol 2018;73:836.

10 D'Souza N, Abulafi M. The faecal immunochemical test in low risk patients with suspected bowel cancer. $\mathrm{Br}$ J Hosp Med 2019;80:22-6. 
11 Chapman C, Bunce J, Oliver S et al. Service evaluation of faecal immunochemical testing and anaemia for risk stratification in the 2-week-wait pathway for colorectal cancer. BJS Open 2019.

12 Nicholson BD, James T, East JE et al. Experience of adopting faecal immunochemical testing to meet the NICE colorectal cancer referral criteria for low-risk symptomatic primary care patients in Oxfordshire, UK. Frontline Gastroenterol 2018.
13 Hippsley-Cox ], Coupland C. Development and validation of risk prediction equations to estimate survival in patients with colorectal cancer: cohort study. BMJ 2017:15:2497.

Address for correspondence: Dr Robert Logan, King's College Hospital, Denmark Hill, London SE5 9RS, UK

Email: robert.logan@nhs.net

\section{NGC

\section{New to systematic reviewing, or need} to improve your critical appraisal skills?

Our intensive 1-day courses will introduce you to the key principles of critical appraisal and systematic reviewing, and equip you with the basic skills to put your knowledge into practice.

$>$ Introduction to critical appraisal

> Systematic review and meta-analysis in action

Discount available if both courses are booked together.

\section{'Informative and practical'}

Dr Rajeswari Ramaraj

For course dates and more information visit: www.rcplondon.ac.uk/ngc-training-courses 